NBER WORKING PAPER SERIES

\title{
OPTIMAL FINES AND AUDITING WHEN WEALTH IS COSTLY TO OBSERVE
}

\author{
A. Mitchell Polinsky \\ Working Paper 10760 \\ http://www.nber.org/papers/w10760 \\ NATIONAL BUREAU OF ECONOMIC RESEARCH \\ 1050 Massachusetts Avenue \\ Cambridge, MA 02138 \\ September 2004
}

This research was supported by the John M. Olin Program in Law and Economics at Stanford Law School. I received helpful comments from Nuno Garoupa, Jason Johnston, Daniel Rubinfeld, Steven Shavell, Kathryn Spier, and Robert Weisberg. The views expressed herein are those of the author(s) and not necessarily those of the National Bureau of Economic Research.

(C2004 by A. Mitchell Polinsky. All rights reserved. Short sections of text, not to exceed two paragraphs, may be quoted without explicit permission provided that full credit, including () notice, is given to the source. 
Optimal Fines and Auditing When Wealth is Costly to Observe

A. Mitchell Polinsky

NBER Working Paper No. 10760

September 2004

JEL No. D31, D62, H23, K14, K42

\begin{abstract}
$\underline{\text { ABSTRACT }}$
This article studies optimal fines when an offender's wealth is private information that can be obtained by the enforcement authority only after a costly audit. I derive the optimal fine for the underlying offense, the optimal fine for misrepresenting one's wealth level, and the optimal audit probability. I demonstrate that the optimal fine for misrepresenting wealth equals the fine for the offense divided by the audit probability, and therefore generally exceeds the fine for the offense. The optimal audit probability is positive, increases as the cost of an audit declines, and equals unity if the cost is sufficiently low. If the optimal audit probability is less than unity, there are some individuals who are capable of paying the fine for the offense who misrepresent their wealth levels. I also show that the optimal fine for the offense results in underdeterrence due to the cost of auditing wealth levels.
\end{abstract}

A Mitchell Polinsky

Stanford Law School

Stanford, CA 94305

and NBER

polinsky@stanford.edu 


\section{Introduction}

Despite the obvious ability of individuals to hide assets (for example, by hoarding cash or distributing property to relatives or friends), prior analyses of optimal fines nearly universally assume that an offender's level of wealth is costlessly observable by the enforcement authority. ${ }^{1}$ The contribution of this article is to consider optimal fines when an offender's wealth is private information that can be obtained by the enforcement authority only after a costly audit.

Obviously, the conventional analysis is a special case of the present analysis, when the audit cost is zero.

I employ a model in which there is a continuum of individuals with respect to their level of wealth and their potential benefit from committing an offense. If an individual commits the offense and is caught, he is sanctioned with a fine. If he claims that he cannot pay the fine, he may be audited. If the audit determines that he misrepresented his wealth level, he can be fined for having lied about his wealth. The enforcement authority's problem is to choose the probability of detecting the offense, the fine for the offense, the probability of an audit, and the fine for misrepresentation of wealth so as to maximize social welfare.

Among other things, I demonstrate that the optimal fine for misrepresenting one's wealth level equals the fine for the offense divided by the audit probability, and therefore generally exceeds the fine for the offense. The optimal audit probability is positive, increases as the cost of an audit declines, and equals unity if the cost is sufficiently low. If the optimal audit probability is less than unity, there are some individuals who are capable of paying the fine for the offense who misrepresent their wealth levels. I also

\footnotetext{
${ }^{1}$ See note 2 below for a discussion of the few exceptions.
} 
show that the optimal fine for the offense results in underdeterrence due to the cost of auditing wealth levels.

Section II presents the model. Section III derives the optimal fines and audit probability. Section IV contains some concluding observations about the use of imprisonment to sanction misrepresentation of wealth and the offense. ${ }^{2}$

\section{Model}

In the model, risk-neutral individuals contemplate whether to commit an offense that causes harm. Each individual is identified by the benefit he would obtain from committing the offense and by his level of wealth. An individual who commits the offense is detected with some probability (which is costly for the state to maintain) and fined. If he claims that he cannot pay the fine because he has insufficient wealth, he may be audited at some cost to the state to determine his wealth level (audits are assumed to be accurate). If an audit reveals that the individual misrepresented his wealth level, he may be sanctioned further, by a higher fine. The fine imposed on an individual, whether for the offense or for misrepresenting his wealth level, cannot exceed his wealth. Fines are assumed to be socially costless to impose.

The following notation will be used.

$h=$ harm caused if the offense is committed; $h>0$;

\footnotetext{
${ }^{2}$ To my knowledge, this article is the first to analyze optimal fines when the offender's level of wealth can be observed by the enforcement authority only after a costly audit. Several studies of related interest should be mentioned, however. Chu and Jiang (1993) and Levitt (1997) consider the choice between fines and imprisonment when wealth cannot be discovered by the enforcement authority at any cost (in Chu and Jiang's case, this assumption is implicit). Garoupa (1998) investigates optimal fines when the enforcement authority is assumed to costlessly observe an underestimate of offenders' wealth levels. See also the discussion of Polinsky (2004) in section IV below. The large theoretical literature on the auditing of wealth levels in the context of controlling tax evasion — see Mookherjee (1997, pp. 207-31) and Andreoni, Erard, and Feinstein (1998, pp. 823-35) - is only tangentially relevant to the analysis here because its focus is on controlling offenses that affect the distribution of
} 
$b=$ benefit from committing the offense; $b \geq 0$;

$$
\begin{aligned}
r(b) & =\text { probability density of } b ; r(b) \text { is positive for all } b \geq 0 ; \\
w & =\text { wealth of an individual; } w \geq 0 ; \\
g(w) & =\text { probability density of } w ; g(w) \text { is positive for all } w \geq 0 ; \\
p & =\text { probability of detection; } \\
e(p) & =\text { enforcement expenditures of the state; } e(0)=0 ; e^{\prime}(p)>0 ; \\
f_{O} & =\text { fine for committing the offense; } f_{O} \geq 0 ;^{3} \\
q & =\text { probability of an audit; } q \geq 0 ; \\
k & =\text { cost to the state of an audit; } k>0 ; \text { and } \\
f_{M} & =\text { fine for misrepresenting one's wealth level; } f_{M} \geq 0 .
\end{aligned}
$$

The distributions of benefits and of wealth are assumed to be independent and known by the state. Without loss of generality, I assume that if an offender is found to have misrepresented his wealth level, the fine $f_{M}$ is imposed instead of the fine $f_{O}$ that otherwise would be applicable.

Behavior of individuals. First consider the decision of an individual who has been caught committing the offense whether to misrepresent his wealth level $w$. If he does not do so, he will pay the fine for the offense $f_{O}$ if $w \geq f_{O}$, and $w$ otherwise. In other words, he will pay $\min \left[w, f_{O}\right]$. If he pays $w$ because his wealth is insufficient to pay $f_{O}$ and he is subsequently audited, he will be found to have not misrepresented his wealth level.

Suppose, however, that the individual does misrepresent his wealth level, claiming he is only capable of paying $w^{\prime}<f_{O}$. If he is not audited, he will pay $w^{\prime}$. If he is audited, he will pay

income rather than that cause harm. Thus, for example, much of this literature is concerned with how auditing policy should be designed and coordinated with tax policy so as to facilitate the raising of revenue.

${ }^{3}$ This is the nominal fine. An individual might not have sufficient wealth to pay this fine. The same statement applies to the fine for misrepresenting one's wealth level. 
$f_{M}$ if he has sufficient wealth to pay this fine; otherwise he will pay his wealth $w$. Thus, if he misrepresents his wealth level, his expected payment is $(1-q) w^{\prime}+q \min \left[w, f_{M}\right]$. If an individual misrepresents his wealth level, he will do so to the greatest extent possible, claiming that he has no wealth. ${ }^{4}$ Hence, his expected payment would be $q \min \left[w, f_{M}\right]$.

Given the preceding discussion, an individual will misrepresent his wealth level if and only if ${ }^{5}$

$$
q \min \left[w, f_{M}\right]<\min \left[w, f_{O}\right] .
$$

Let

$\hat{w}=$ critical level of wealth below which individuals who are detected committing the offense will misrepresent their wealth levels and at and above which individuals will not misrepresent their wealth levels.

It can be demonstrated from (1) that

$$
\hat{w}= \begin{cases}f_{O} / q & \text { if } q f_{M} \geq f_{O} \\ \infty & \text { if } q f_{M}<f_{O}{ }^{6}\end{cases}
$$

In other words, if the expected fine for misrepresenting one's wealth level equals or exceeds the fine for the offense, individuals with wealth less than $f_{o} / q$ will misrepresent their wealth levels

\footnotetext{
${ }^{4}$ This occurs because the fine for misrepresentation is assumed not to depend on the extent of misrepresentation and the probability of being audited is assumed not to depend on the reported wealth level. The analysis would be much more complicated if these assumptions were not made, because it would become necessary to choose the optimal schedules for the fine for misrepresentation and the audit probability, as well as to derive the behavior of individuals with different levels of wealth in response to these schedules.

${ }^{5}$ It is convenient to assume that if an individual is indifferent between misrepresenting his wealth level and not, he does not misrepresent his wealth level. None of the results depend on this assumption.

${ }^{6}$ To prove this requires evaluating (1) in three cases - whether $q f_{M}$ exceeds, equals, or is less than $f_{O}$. Because a complete treatment of these cases is tedious, I consider only one here. Suppose that $q f_{M}>f_{O}$. In this case, $f_{O}<f_{O} / q<f_{M}$. If $w \leq f_{O}$, (1) becomes $q w<w$, which clearly holds. If $f_{O}<w<f_{O} / q$, (1) becomes $q w<f_{O}$, which holds because $w<f_{O} / q$. If $f_{O} / q \leq w<f_{M}$, (1) becomes $q w<f_{O}$, which does not hold because $f_{O} / q \leq w$. If $w \geq f_{M}$, (1) becomes $q f_{M}<f_{O}$, which does not hold because $f_{O} / q<f_{M}$. Hence, if $q f_{M}>f_{O}$, individuals with wealth levels less than $f_{O} / q$ will misrepresent their wealth levels, while individuals with wealth levels equal to or greater than $f_{O} / q$ will not.
} 
(claiming to be zero-wealth individuals), while individuals with wealth equal to or greater than $f_{O} / q$ will pay the fine for the offense $f_{O}$. Note that if $q<1$, there are some individuals who are capable of paying $f_{O}$ who choose to misrepresent their wealth levels (individuals with wealth between $f_{O}$ and $\left.f_{O} / q\right)^{7}$ If, however, the expected fine for misrepresenting one's wealth level is less than the fine for the offense, all individuals will misrepresent their wealth levels.

Next consider the decision of an individual whether to commit the offense. Let $b(w)=$ critical value of benefit below which an individual whose wealth is $w$ will not commit the offense and at and above which he will. ${ }^{8}$

Thus, the higher $b(w)$ is, the greater the level of deterrence.

An individual will commit the offense if his benefit equals or exceeds the expected fine he faces, which depends on whether he will misrepresent his wealth level if he is caught. Since he will misrepresent his wealth if and only if (1) holds, it follows that the critical value of benefit is

$$
b(w)=p \min \left\{q \min \left[w, f_{M}\right], \min \left[w, f_{O}\right]\right\} .
$$

Social welfare. Social welfare is the sum of the benefits obtained by individuals who commit the offense, less the harm done, less the cost of detection, and less the cost of auditing. Thus, social welfare is

$$
\begin{aligned}
& \int_{0}^{\hat{w}}\left\{\int_{b(w)}^{\infty}[b-h-p q k] r(b) d b\right\} g(w) d w \\
& +\int_{\hat{w}}^{\infty}\left\{\int_{b(w)}^{\infty}[b-h] r(b) d b\right\} g(w) d w-e(p),
\end{aligned}
$$

\footnotetext{
${ }^{7}$ Individuals whose wealth is less than $f_{O}$ obviously will not pay anything because the worst that can happen to them is that they are audited and lose their wealth.

${ }^{8}$ I assume without loss of generality that an individual commits the offense if he is indifferent.
} 
where $b(w)$ is given by (3) and $\hat{w}$ by (2). The first term in (4) is the contribution to social welfare associated with individuals with relatively low levels of wealth (less than $\hat{w}$ ) who misrepresent their wealth levels and do not pay the fine for the offense $f_{O}$. Every individual in this group whose benefit equals or exceeds $b(w)$ commits the offense, resulting in a benefit $b$, a harm $h$, and an expected auditing cost of $p q k$. The second term is the contribution to social welfare associated with relatively high-wealth individuals who pay the fine for the offense $f_{O}$. The state also incurs enforcement expenditures $e$.

The state's problem. The state's problem is to maximize social welfare through the choice of the probability of detection $p$, the fine for the offense $f_{O}$, the audit probability $q$, and the fine for misrepresenting one's wealth level $f_{M}{ }^{9}$ The fines actually paid by individuals cannot exceed their levels of wealth. Asterisks will be used to denote the solution to the state's problem, which I assume is unique.

Because the choice of the optimal probability of detection $p^{*}$ does not bear in any interesting way on the analysis of optimal fines and auditing, I will not analyze the choice of $p^{*}$ below. However, I assume that some enforcement is optimal, that is, $p^{*}>0$; otherwise, the problem is uninteresting.

\section{Analysis}

In this section I describe the optimal enforcement system through four propositions. Following the proof of each proposition is a brief informal discussion of the result. ${ }^{10}$

\footnotetext{
${ }^{9}$ Because my objective is to derive the socially optimal enforcement policy, I do not consider whether the state's policy is credible.

${ }^{10}$ Although some of the proofs convey useful intuition, they may be skipped with little loss of continuity.
} 
Propositions 1 and 2 describe the optimal fine for misrepresenting wealth, and its effect on individuals' decisions to engage in misrepresentation, when the audit probability is, respectively, less than or equal to unity. Proposition 3 characterizes the optimal fine for the offense. Proposition 4 establishes that the optimal audit probability is positive and may be less than or equal to unity.

Proposition 1. If the optimal audit probability is less than unity, $q^{*}<1$, then:

(a) the optimal fine for misrepresenting one's wealth level $f_{M}^{*}$ exceeds the optimal fine for the offense; in particular, it equals the fine for the offense divided by the audit probability: $f_{M}^{*}=$ $f * q^{*}>f \mathscr{\delta} ;{ }^{11}$

(b) individuals with wealth below the fine for misrepresentation $f_{M}^{*}$ misrepresent their

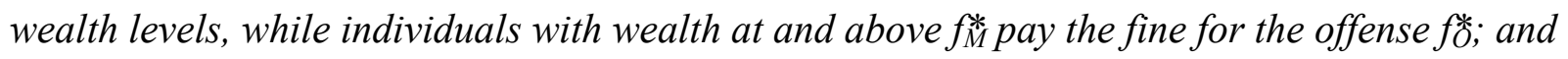
(c) there are some individuals who are capable of paying the fine for the offense fơ who misrepresent their wealth levels (individuals with wealth between $f^{*}$ and $f_{M}^{*}$ ).

Proof: (i) I first show that the optimal enforcement system is characterized by $q f_{M} \geq f_{O}$. Assume otherwise, that $q f_{M}<f_{O}$. If this condition were to hold, then individuals would misrepresent their wealth regardless of their level of wealth (see (2)). Consequently, everyone will be audited with probability $q$ and their expected fine, conditional on having been detected committing the offense, will be $q w$ for $w<f_{M}$ and $q f_{M}$ for $w \geq f_{M}$. It is possible, however, to raise social welfare by lowering $f_{O}$ to $q f_{M}$. If $f_{O}=q f_{M}$, then $f_{O}<f_{M}$. If $w \leq f_{O}$, (1) becomes $q w<w$, which holds. If $f_{O}<w<f_{M}$, (1) becomes $q w<f_{O}$, which holds because $f_{O}=q f_{M}$ and $w<f_{M}$. If $w$ $\geq f_{M}$, (1) becomes $q f_{M}<f_{O}$, which does not hold because $f_{O}=q f_{M}$. Thus, if $f_{O}$ is lowered to $q f_{M}$,

\footnotetext{
${ }^{11}$ As will be seen, $f_{M}^{*}$ is not unique. Any $f_{M}$ equal to or greater than $f_{\delta}^{*} / q^{*}$ is optimal. An analogous observation applies to Proposition 2 below.
} 
individuals for whom $w<f_{M}$ will continue to misrepresent their wealth levels, be audited with probability $q$, and face an expected fine conditional on detection of $q w$. But individuals for whom $w \geq f_{M}$ will now choose to pay the fine for the offense $f_{O}$ and not misrepresent their wealth levels. Since $f_{O}=q f_{M}$, they are deterred to the same extent as before. But since they pay the fine $f_{O}$, they are not audited. Thus, the behavior of individuals is not affected, but auditing costs are reduced, thereby raising social welfare. In the optimal enforcement system, therefore, it must be that $q f_{M} \geq f_{O}$.

(ii) I next show that in the optimal enforcement system one can assume without loss of generality that $q f_{M}=f_{O}$. Since step (i) rules out $q f_{M}<f_{O}$, suppose $q f_{M}>f_{O}$. Then, by (2), individuals for whom $w<f_{o} / q$ would misrepresent their wealth levels and individuals for whom $w \geq f_{O} / q$ would not misrepresent their wealth. Consequently, an individual in the former group will be audited with probability $q$ and his expected fine, conditional on detection, will be $q w$ (he is unable to pay $f_{M}$ since $w<f_{O} / q<f_{M}$ ). Individuals in the latter group will pay the fine for the offense $f_{O}$ (they are able to because $w \geq f_{O} / q>f_{O}$ ) and not be audited. If $f_{M}$ is lowered until $q f_{M}=$ $f_{0}$, it is easy to see that individuals' decisions about misrepresenting their wealth levels, and their expected fines, are not affected. Hence, $f_{M}$ such that $q f_{M}=f_{O}$ is optimal (as is any higher $f_{M}$ ), establishing part (a) of the proposition.

(iii) Given $q f_{M}=f_{O}$, (2) implies that $\hat{w}=f_{O} / q=f_{M}$. In other words, individuals with wealth below $f_{M}$ misrepresent their wealth levels, while individuals with wealth at or above $f_{M}$ do not. Since $f_{M}=f_{O} / q>f_{O}$, the latter individuals pay the fine $f_{O}$. This establishes part (b).

(iv) Part (c) follows immediately from step (iii) since individuals with wealth between $f_{O}$ and $f_{M}=f_{O} / q>f_{O}$ misrepresent their wealth levels. 
Comment: It is easy to see why the optimal fine for misrepresenting one's wealth level $f_{M}^{*}$ must be at least equal to the fine for the offense divided by the audit probability, $f{ }^{*} / q^{*}$. If this were not the case, then the expected fine for misrepresenting one's wealth level, $q^{*} f_{M}^{*}$, would be less than the fine for the offense, $f$, , and no one would pay the fine for the offense. To induce individuals to pay the fine for the offense, the fine for misrepresentation must be at least equal to $f^{*} / q^{*}$. Any higher fine for misrepresentation has the same effect. Even if $f_{M}^{*}=f^{*} / q^{*}$, not everyone will pay the fine for the offense. In particular, individuals whose wealth is below $f_{M}^{*}$ are unable to pay the full fine for misrepresentation, and thus cannot be induced to pay the fine for the offense; since $f_{M}^{*}>f \frac{*}{\delta}$, this includes some individuals who are capable of paying $f f_{\delta}^{*}$. But everyone else can be threatened with a fine for misrepresentation of $f_{M}^{*}$ and therefore will pay the fine for the offense.

Proposition 2. If the optimal audit probability equals unity, $q^{*}=1$, then:

(a) the optimal fine for misrepresenting one's wealth level equals the optimal fine for the offense: $f_{M}^{*}=f$;

(b) individuals do not misrepresent their wealth levels; and

(c) everyone who is able to pay the fine for the offense fơ⿱宀㠯 does so.

Proof: The proof of part (a) parallels steps (i) and (ii) in the proof of Proposition 1. Part (b) follows from (1) given $f_{M}=f_{O}$. Part (c) follows immediately from part (b)

Comment: Clearly, if an audit is certain if one does not pay the fine for the offense, then a fine for misrepresentation equal to the fine for the offense will induce those who are able to pay the fine for the offense to do so. Individuals who are unable to pay the fine for the offense do not have an incentive to misrepresent their wealth levels since they will have to pay their wealth whether they are subject to the fine for the offense or the fine for misrepresentation. 


\section{Proposition 3. The optimal fine for the offense fơ}

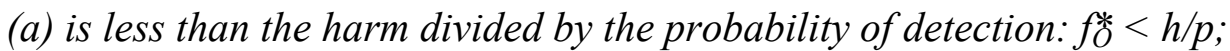

(b) results in underdeterrence due to the cost of auditing; and

(c) declines as the cost of auditing $k$ increases.

Proof: (i) To show that $f\left(\frac{*}{\delta}<h / p\right.$, first rewrite social welfare (4) using the result from part (a) of Proposition 1 that $q f_{M}=f_{O}$. It follows from (2) that $\hat{w}=f_{O} / q$. For $w \leq f_{O} / q=f_{M}$, it follows from (3) that $b(w)=p \min \left\{q w, \min \left[w, f_{O}\right]\right\}=p q w$. For $w>f_{O} / q=f_{M}, b(w)=p \min \left\{q f_{M}, f_{O}\right\}=$ $p f_{o}$. Thus, social welfare can be rewritten as:

$$
\int_{0}^{f_{O} / q}\left\{\int_{p q w}^{\infty}[b-h-p q k] r(b) d b\right\} g(w) d w
$$

From (5), the first-order condition with respect to the fine for the offense $f_{O}$ is:

$$
-p q k\left[1-R\left(p f_{O}\right)\right]\left[d G\left(f_{O} / q\right) / d f_{O}\right]-\left[p f_{O}-h\right]\left[1-G\left(f_{O} / q\right)\right]\left[d R\left(p f_{O}\right) / d f_{O}\right]=0,
$$

where $G($.$) is the cumulative distribution of wealth and R($.$) is the cumulative distribution of$ benefits. Since the first term in (6) is negative, the second term must be positive, which requires $p f_{O}-h$ to be negative. In other words, it must be that $f_{O}<h / p$, establishing part (a).

(ii) If auditing were costless, $k=0$, (6) would require that $p f_{O}-h=0$, or $p f_{O}=h$; since the expected fine for the offense equals the harm, this would result in first-best deterrence (for everyone who pays $f_{O}$ ). But if auditing is costly, $k>0$, step (i) showed that $p f_{O}<h$, resulting in underdeterrence. This establishes part (b).

(iii) By the implicit function theorem and the assumption that the second-order condition for a maximum is satisfied with respect to the fine for the offense $f_{O}$, the sign of $d f_{O} / d k$ is the 
same as the sign of the derivative of the left-hand side of (6) with respect to $k$. The latter derivative is $-p q\left[1-R\left(p f_{O}\right)\right]\left[d G\left(f_{O} / q\right) / d f_{O}\right]<0$. Thus, $d f_{O} / d k<0$, proving part (c).

Comment: To achieve first-best deterrence, the expected fine for the offense $p f_{O}$ would have to equal the harm $h$ or, equivalently, the fine for the offense $f_{O}$ would have to equal $h / p$. The reason it is optimal to employ a lower fine, and tolerate some underdeterrence, is that doing so reduces auditing costs. Specifically, if $f_{O}$ is lowered, more individuals will be willing to pay $f_{O}$ rather than misrepresent their wealth levels (Proposition 1 established that individuals with wealth levels above $f_{M}=f_{O} / q$ will pay the fine for the offense). These individuals no longer will be audited, thereby saving auditing costs. There is no first-order reduction in social welfare due to the marginal individuals who now commit the offense as a result of the lower fine for the offense, because they were individuals whose benefits just equaled the harm. Thus, it is optimal to set the fine for the offense $f_{O}$ such that there is some underdeterrence, in order to reduce auditing costs. Obviously, the higher the cost of an audit, the greater the motive to lower $f_{O}$ for this reason.

Note that, although a fine usually is treated as a socially costless sanction because it is a mere transfer of wealth, it is no longer socially costless if auditing is required to induce individuals to pay the fine. In effect, there is a marginal social cost incurred from imposing a higher fine due to the need for a higher audit rate to induce the payment of the fine. As a consequence, the fine for the offense should not be as high as it would be in the absence of auditing costs. $^{12}$

\footnotetext{
12 The results in Polinsky and Shavell (1991), where it was assumed that offenders' wealth levels could be observed without cost, are a special case of the present analysis - when the cost of an audit, $k$, is zero. There it was shown that the optimal fine for the offense is $h / p$, implying that individuals with wealth levels equal to or greater than $h / p$ are deterred to the first-best extent. Here, due to the cost of auditing, the optimal fine is less than $h / p$, implying that all individuals are underdeterred.
} 


\section{Proposition 4. The optimal audit probability $q^{*}$}

(a) is positive: $q^{*}>0$;

(b) rises as the cost of auditing $k$ declines; and

(c) equals unity for $k$ sufficiently low.

Proof: (i) To demonstrate that the optimal audit probability $q^{*}$ is positive, suppose it were zero. Then everyone would misrepresent their wealth levels. The critical value of benefit $b(w)$ would be zero for all wealth levels, regardless of the probability of detection $p$ (see (3)). But then it would be optimal for the state to spend nothing on enforcement, contradicting the assumption that $p^{*}>0$. Thus, if the offense is worth deterring to any extent - that is, if $p^{*}>$ 0 - it must be that $q^{*}$ is positive, establishing part (a). ${ }^{13}$

(ii) To see that $q^{*}$ rises as $k$ declines, consider the first-order condition with respect to $q$; using (5), and after some manipulation, it can be written as:

$$
\begin{gathered}
k\left(-\left\{\int_{p f_{0}}^{\infty} p r(b) d b\right\}\left[d G\left(f_{o} / q\right) / d q\right]+\int_{0}^{f_{o} / q}\left\{[p q][d R(p q w) / d q]-\int_{p q w}^{\infty} p r(b) d b\right\} g(w) d w\right) \\
-\int_{0}^{f_{o} / q}\{[p q w-h][d R(p q w) / d q]\} g(w) d w=0 .
\end{gathered}
$$

By the implicit function theorem and the assumption that the second-order condition for a maximum is satisfied with respect to $q$, the sign of $d q / d k$ is the same as the sign of the derivative of (7) with respect to $k$. The latter derivative is the expression in large brackets in (7). To see that this expression must be negative, observe that $p q w-h<0$ for $w \leq f_{O} / q$ because, by part (a)

\footnotetext{
${ }^{13}$ This result does not require that the lowest level of wealth is zero, only that it is sufficiently low. Even if the state could impose a fine for the offense equal to the lowest level of wealth without auditing individuals, if this level is sufficiently low, the value of the resulting deterrence will be less than the cost of detection.
} 
of Proposition 3, $f_{o} / q<h / p q$. Thus, the last term in (7) is positive, which implies that the expression in large brackets must be negative. This proves part (b).

(iii) To see that $q^{*}=1$ for $k$ sufficiently low, I will first show that if $k=0, q^{*}=1$. If $k=$ 0 , the derivative of social welfare with respect to $q$ evaluated at $q=1$ is the second term on the left-hand side of (7) evaluated at $q=1$, which is

$$
-\int_{0}^{f_{O}}\{[p w-h][d R(p w) / d q]\} g(w) d w .
$$

If $k=0$, step (ii) of the proof of Proposition 3 showed that $f_{O}=h / p$. Since $p w-h<0$ for all $w<$ $h / p,(8)$ is positive. This implies that $q^{*}=1$ if $k=0$. Clearly, if $k$ is sufficiently small, the derivative of social welfare with respect to $q$ evaluated at $q=1$ will continue to be positive, in which case $q^{*}$ will continue to equal unity. This establishes part (c).•

Comment: If it is optimal to expend resources on trying to detect offenders, it must be optimal to audit them with some probability if they are caught, since otherwise the expenditures on detection would be wasted. (Of course, it might be optimal not to deter the harmful activity at all if the cost of detection and/or the cost of auditing is sufficiently great.) For any given probability of detection, a higher audit probability is beneficial because it induces more offenders to pay the fine for the offense $f_{O}$ rather than misrepresent their wealth levels (see parts (a) and (b) of Proposition 1), and thereby deters them to a better degree. Thus, if the cost of auditing were to decline, the optimal audit probability would rise, and if the cost were low enough, the optimal audit probability would be unity. 


\section{Concluding Remarks}

Although this article has considered monetary sanctions only, I want to observe in closing that the appropriate use of an imprisonment sanction for misrepresenting one's wealth could increase social welfare. Specifically, suppose an imprisonment sentence, in addition to the fine $f_{M}$, is imposed on individuals who are found to have been capable of paying the fine for the offense $f_{O}$ but who nonetheless misrepresented their wealth level. (Individuals who are not able to pay the fine $f_{O}$ are sanctioned only with a fine equal to their wealth if they are found to have misrepresented their wealth level, as above.) If the imprisonment sentence is high enough, such individuals now can be induced to pay $f_{O}$ instead of misrepresenting their wealth level; and since they pay $f_{O}$, they will not be audited and will not bear the sentence. The behavior of all other individuals is unaffected under this sentencing policy. Hence, social welfare rises because the extent of underdeterrence, as well as the cost of auditing, declines for the affected individuals.

The preceding paragraph establishes that some use of the threat of imprisonment to deter misrepresentation of wealth is socially desirable, but it does not derive the optimal use of imprisonment sanctions for this purpose. It may be optimal also to impose imprisonment sentences for misrepresentation on individuals who are not capable of paying the fine for the offense $f_{O}$. Although some of these individuals will be audited and bear the imprisonment sentence, the additional deterrence due to use of an imprisonment sanction can allow the auditing probability $q$ or the probability of detection $p$ to be lowered, thereby saving auditing or enforcement costs. Thus, the optimal use of imprisonment to sanction misrepresentation of wealth might involve sentences that depend on whether an individual is found to have been capable of paying the fine for the offense $f_{O}$. 
Another possibility is to use imprisonment to sanction the offense. In a companion paper - Polinsky (2004) - I consider the optimal use of fines and imprisonment when wealth is assumed to be impossible to observe. I demonstrate in the model employed there that when imprisonment sentences are used, the optimal choice of sanctions induces high-wealth offenders to pay a fine that exceeds the wealth level of low-wealth offenders; by paying the fine, they avoid a less desirable combination of sanctions they would bear if they paid only as much as low-wealth offenders and faced a longer imprisonment sentence. ${ }^{14}$ Thus, an appropriately designed sanctioning policy that includes imprisonment sentences for the offense can serve as an alternative to auditing — both can reduce the incentive of offenders to misrepresent their wealth levels.

${ }^{14}$ This result is similar in spirit to a point developed by Levitt (1997), though he formally considers fines and imprisonment sentences as alternatives, rather than the optimal combination of the sanctions. 


\section{References}

Andreoni, James, Brian Erard, and Jonathan Feinstein, “Tax Compliance,” Journal of Economic Literature, Vol. 36, No. 2 (June 1998), pp. 818-60.

Chu, C. Y. Cyrus, and Neville Jiang “Are Fines More Efficient than Imprisonment?” Journal of Public Economics, Vol. 51, No. 3 (July 1993), pp. 391-413.

Garoupa, Nuno, “Optimal Law Enforcement and Imperfect Information when Wealth Varies Among Individuals," Economica, Vol. 65 (1998), pp. 479-90.

Levitt, Steven, "Incentive Compatibility Constraints as an Explanation for the Use of Prison Sentences Instead of Fines," International Review of Law and Economics, Vol. 17, No. 2 (June 1997), pp. 179-92.

Mookherjee, Dilip, “The Economics of Enforcement," in Amitava Bose, Mihir Rakshit and Anup Sinha (Editors), Issues in Economic Theory and Policy, Essays in Honor of Tapas Majumdar (New Delhi: Oxford University Press, 1997), pp. 202-49.

Polinsky, A. Mitchell, “The Optimal Use of Fines and Imprisonment When Wealth is Unobservable,” Working Paper No. 287, John M. Olin Program in Law and Economics, Stanford Law School, August 2004.

Polinsky, A. Mitchell, and Steven Shavell, “A Note on Optimal Fines When Wealth Varies Among Individuals," American Economic Review, Vol. 81, No. 3 (June 1991), pp. 618-21. 